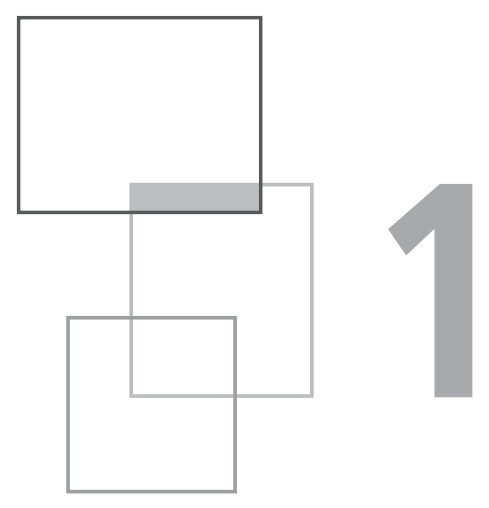

\title{
Les fondements
}

\section{d'une vision énergétique raisonnée}

La politique de l'énergie est, dans les sociétés modernes, d'une importance capitale car elle conditionne largement notre mode de vie et la qualité de celle-ci. Elle est orientée par les Autorités responsables de l'État mais très largement conduite par les grandes entreprises du secteur, qu'elles soient nationales ou privées, dans le cadre des réglementations françaises et européennes.

Les objectifs visés par une politique énergétique doivent émerger d'une vision à long terme qui tient compte à la fois de l'état actuel du pays et du monde, et des perspectives d'un avenir dans lequel notre pays prendra place dans son meilleur intérêt. Cette vision du futur énergétique s'élabore dans un champ de contraintes de différentes natures :

- les règles internationales en la matière (règles du commerce mondial et réglementation européenne) ;

- les contraintes techniques et économiques déterminant le coût de l'énergie fournie à l'utilisateur ;

- la situation politique nationale et internationale, conditionnant la sécurité d'approvisionnement et l'indépendance nationale vis-à-vis des sources de combustibles ;

- les exigences sociales, environnementales et de santé publique. 


\subsection{La situation actuelle}

La situation actuelle de la France résulte d'une volonté politique, forte et constante, de recherche d'un niveau d'indépendance énergétique acceptable, assurant une fourniture régulière. Elle a été soutenue par les gouvernements successifs du pays depuis la fin de la guerre en 1945.

Disposant de peu de ressources pétrolières, la France, jusqu'aux années soixante, a maintenu un taux d'indépendance satisfaisant, en s'appuyant essentiellement sur les productions nationales hydrauliques (avec des investissements considérables), et la poursuite de l'exploitation du charbon et la biomasse.

Dès les années soixante, la consommation d'énergie s'est envolée, avec le développement économique des trente glorieuses et l'amélioration, considérable, du niveau de vie. Les incertitudes sur l'approvisionnement en pétrole, provenant du Moyen-Orient mais aussi d'une Algérie devenue indépendante, ont conduit à un développement de l'exploitation du gaz (gaz de Lacq), pourtant d'exploitation difficile.

Au début des années soixante-dix, alors que la consommation poursuivait sa croissance (de 85 millions de tep en 1960 à 149 en 1970), survenait le premier choc pétrolier (1973).

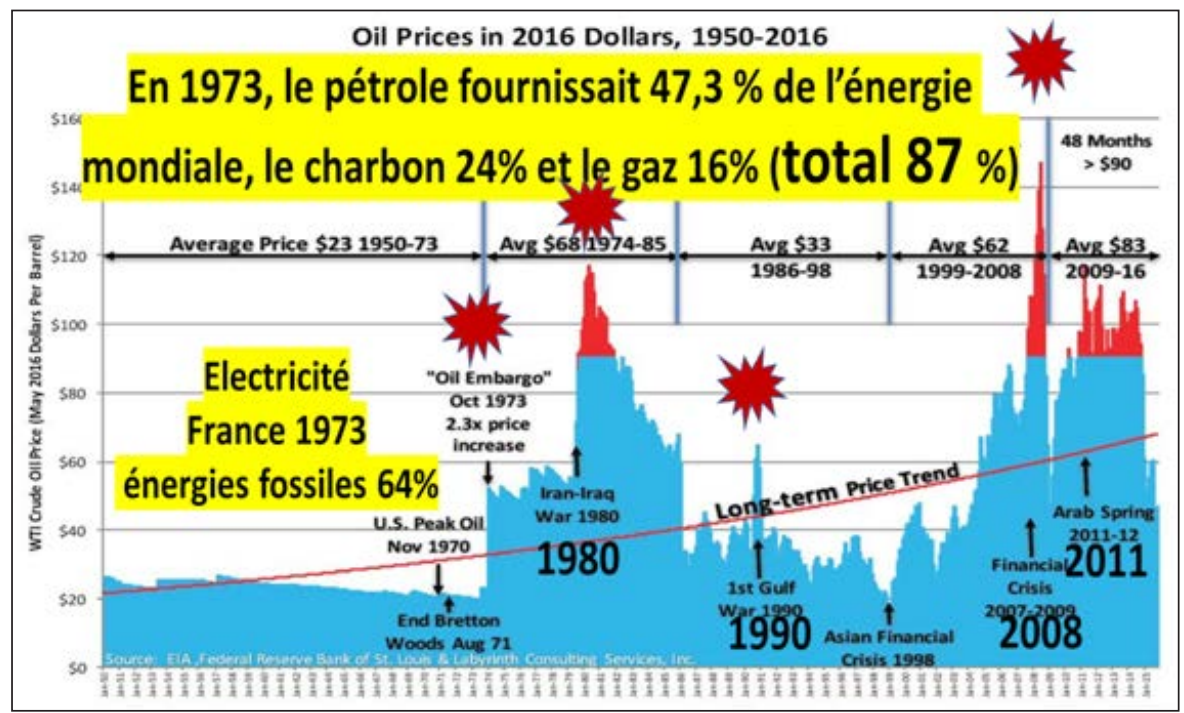

Figure 1 Évolution du prix du pétrole en \$/baril constants de 1950 à 2015 : de la stabilité à l'instabilité. 
C'était le début d'une longue succession d'incertitudes sur l'approvisionnement mondial d'énergies fossiles. Dans le même temps, la dépendance du pays au pétrole, puis au gaz, augmentait très rapidement (pour l'industrie, le chauffage et la mobilité en particulier). Et nos réserves de charbon et de gaz s'amenuisaient. Le pétrole devenait très prépondérant, $62 \%$ de notre énergie en 1975.

Tableau 1 Évolution de notre consommation d'énergie primaire de 1960 à 1975 en Mtep.

\begin{tabular}{|l|c|c|c|c|c|c|}
\cline { 2 - 7 } \multicolumn{1}{c|}{} & $\mathbf{1 9 6 0}$ & $\mathbf{1 9 6 5}$ & $\mathbf{1 9 7 0}$ & $\mathbf{1 9 7 3}$ & $\mathbf{1 9 7 4}$ & $\mathbf{1 9 7 5}$ \\
\hline Pétrole & $\begin{array}{c}26,9 \\
(31,4 \%)\end{array}$ & $\begin{array}{c}49,7 \\
(44,7 \%)\end{array}$ & $\begin{array}{c}87,3 \\
(58,9 \%)\end{array}$ & $\begin{array}{c}116,3 \\
(66,5 \%)\end{array}$ & $\begin{array}{c}112,4 \\
(64,0 \%)\end{array}$ & $\begin{array}{c}101,7 \\
(61,9 \%)\end{array}$ \\
\hline Charbon & $\begin{array}{c}46,8 \\
(54,6 \%)\end{array}$ & $\begin{array}{c}45,7 \\
(41,0 \%)\end{array}$ & $\begin{array}{c}38,1 \\
(25,6 \%)\end{array}$ & $\begin{array}{c}30,50 \\
(17,4 \%)\end{array}$ & $\begin{array}{c}31,60 \\
(18,0 \%)\end{array}$ & $\begin{array}{c}27,50 \\
(16,7 \%)\end{array}$ \\
\hline Gaz naturel & $\begin{array}{c}2,90 \\
(3,3 \%)\end{array}$ & $\begin{array}{c}5,10 \\
(4,5 \%)\end{array}$ & $\begin{array}{c}9,30 \\
(6,2 \%)\end{array}$ & $\begin{array}{c}15,00 \\
(8,5 \%)\end{array}$ & $\begin{array}{c}16,00 \\
(9,1 \%)\end{array}$ & $\begin{array}{c}17,50 \\
(10,6 \%)\end{array}$ \\
\hline Hydraulique & $9,00(10,5)$ & $\begin{array}{c}10,5 \\
(10,5 \%)\end{array}$ & $\begin{array}{c}12,4 \\
(18,3 \%)\end{array}$ & $\begin{array}{c}10,00 \\
(5,7 \%)\end{array}$ & $\begin{array}{c}12,60 \\
(7,2 \%)\end{array}$ & $\begin{array}{c}13,50 \\
(8,2 \%)\end{array}$ \\
\hline Nucléaire & - & $\begin{array}{c}0,20 \\
(0,17 \%)\end{array}$ & $\begin{array}{c}1,20 \\
(0,80 \%)\end{array}$ & $\begin{array}{c}3,00 \\
(1,70 \%)\end{array}$ & $\begin{array}{c}3,00 \\
(1,70 \%)\end{array}$ & $\begin{array}{c}4,20 \\
(2,60 \%)\end{array}$ \\
\hline Total & $\mathbf{8 5 , 6}$ & $\mathbf{1 1 1 , 2}$ & $\mathbf{1 4 8 , 3}$ & $\mathbf{1 7 4 , 8}$ & $\mathbf{1 7 5 , 6}$ & $\mathbf{1 6 4 , 4}$ \\
\hline
\end{tabular}

Le gouvernement décidait alors, en 1973, d'agir dans trois directions principales : le développement du nucléaire pour la production d'électricité, l'efficacité énergétique (la lutte contre le « Gaspi »), et le recours au chauffage électrique.

À partir du début des années quatre-vingt-dix, et en particulier du Protocole de Kyoto en 1997 (COP 3), apparaissent des préoccupations environnementales croissantes, notamment en ce qui concerne le risque de réchauffement climatique lié à l'accumulation dans l'atmosphère de gaz à effet de serre (GES), mais également la pollution atmosphérique. Un bouleversement du contexte énergétique en résulte : le concept d'énergies peu ou pas carbonées s'impose. La France engage dans les années 2005/2015 un développement volontariste des énergies renouvelables, quelques années après l'Allemagne. Sont particulièrement privilégiés l'éolien, le solaire et les bioénergies, nos capacités de développement de l'hydroélectricité étant devenues limitées. Ces stratégies dépassent maintenant le plan national pour s'inscrire dans le cadre de l'Union européenne. 
Aujourd'hui on peut afficher pour la France le bilan suivant :

1. La consommation annuelle des différentes sources d'énergie selon les usages

Tableau 2 Bilan de la consommation d'énergie finale en Mtep en France en $2015^{96}$.

\begin{tabular}{|l|c|c|c|c|c|c|c|}
\cline { 2 - 8 } & Charbon & Pétrole & Gaz & Électricité & $\begin{array}{c}\text { Renouvelables } \\
\text { thermiques }\end{array}$ & TOTAL & $\begin{array}{c}\% \text { du } \\
\text { total }\end{array}$ \\
\hline Industrie & 4,9 & 2,1 & 9,7 & 10 & 1,7 & $\mathbf{2 8 , 4}$ & 19 \\
\hline $\begin{array}{l}\text { Résidentiel } \\
\text { et tertiaire }\end{array}$ & 0,3 & 9,9 & 20,4 & 25,7 & 10,6 & $\mathbf{6 6 , 9}$ & 45,6 \\
\hline Agriculture & & 3,3 & 0,3 & 0,7 & 0,2 & $\mathbf{4 , 5 0}$ & 3 \\
\hline Transports & & 45,4 & 0,1 & 0,9 & 3 & $\mathbf{4 9 , 4}$ & 33 \\
\hline TOTAL & $\mathbf{5 , 2}$ & $\mathbf{6 0 , 8}$ & $\mathbf{3 0 , 5}$ & $\mathbf{3 7 , 2}$ & $\mathbf{1 5 , 5}$ & $\mathbf{1 4 9 , 2}$ & $\mathbf{1 0 0}$ \\
\hline
\end{tabular}

Pour les deux tiers, notre consommation finale d'énergie repose sur les combustibles fossiles, importés en quasi-totalité. L'électricité reste le principal vecteur permettant de réduire cette dépendance et de diminuer les émissions de gaz à effet de serre, grâce à l'utilisation de l'énergie nucléaire et des énergies renouvelables.

\section{La production brute d'électricité par sources}

Tableau 3 Production d'électricité en TWh en France en $2015^{97}$.

\begin{tabular}{|l|c|c|c|c|}
\cline { 2 - 4 } \multicolumn{1}{c|}{} & $\begin{array}{c}\text { Énergie } \\
\text { nucléaire }\end{array}$ & $\begin{array}{c}\text { Énergies } \\
\text { renouvelables* } \\
\text { (dont hydraulique) }\end{array}$ & $\begin{array}{c}\text { Thermique } \\
\text { classique** (majorité } \\
\text { combustibles fossiles) }\end{array}$ & TOTAL \\
\hline $\begin{array}{l}\text { Production } \\
\text { (brute en TWh) }\end{array}$ & 437 & $89,5(60,9)$ & 41,5 & $\mathbf{5 6 8}$ \\
\hline $\begin{array}{l}\text { Part de la production } \\
\text { totale (\%) }\end{array}$ & 76,9 & $15,8(10,7)$ & 7,3 & 100 \\
\hline
\end{tabular}

(*) : Hydraulique fil de l'eau, hydraulique de barrage, Station de transfert d'énergie par pompage (STEP) et électricité à partir de déchets renouvelables, de biomasse solide et de biogaz.

(**): Charbon, pétrole, gaz, déchets non renouvelables.

96. D'après le ministère de l'Environnement, de l'Énergie et de la Mer/Service de l'observation et des statistiques (document 2015) pour les tableaux 2 et 3 ; les consommations sont ici corrigées des variations climatiques.

97. http://www.rte-france.com/sites/default/files/2015_bilan_electrique.pdf 
La stratégie mise en œuvre d'un important programme électronucléaire s'est révélée particulièrement positive selon tous les critères mentionnés :

- indépendance nationale : le combustible nucléaire, bien qu'importé, provient de sources diversifiées et peut se stocker sans difficultés pour couvrir les besoins de plusieurs années. La variabilité du coût de l'uranium naturel, qui ne représente que 7 à $8 \%$ du coût de production de l'électricité, ne présente pas de risque ;

- coût de production du $\mathrm{kWh}$ : il s'avère particulièrement compétitif, actuellement 40 à $50 € / M W h$ pour les centrales nucléaires (rapports de la Cour des comptes ${ }^{98}$ );

- émissions de gaz à effet de serre du secteur parmi les plus faibles des pays développés :

- en France, $388 \mathrm{Mt}$ eq. $\mathrm{CO}_{2}$ émis en 2015 (5,8 t eq. $\mathrm{CO}_{2} / \mathrm{an} / \mathrm{hab}$.) dont seulement 23,1 Mt eq. $\mathrm{CO}_{2}$ pour la production électrique, selon RTE, celle-ci étant décarbonée à plus de $93 \%$, soit 42,3 g eq. $\mathrm{CO}_{2} / \mathrm{kWh}$;

- en Allemagne, souvent citée, à tort, comme modèle, $925 \mathrm{Mt}$ eq. $\mathrm{CO}_{2}$ émis $\left(11,1 \mathrm{t}\right.$ eq. $\mathrm{CO}_{2} / \mathrm{an} / \mathrm{hab}$.) dont $308 \mathrm{Mt}$ eq. $\mathrm{CO}_{2}$ pour la production électrique, soit $480 \mathrm{~g}$ eq. $\mathrm{CO}_{2} / \mathrm{kWh}$.

\subsection{Les évolutions en cours}

Partant de l'état décrit ci-dessus, les orientations de la politique énergétique des prochaines années se trouvent déjà encadrées :

- par les dispositions légales adoptées sur le plan national :

- les deux lois «Grenelle 1 " et "Grenelle 2 " de l'environnement de 2009 et 2010 ;

- puis la loi de Transition énergétique pour une croissance verte (LTECV) de 2015 et sa déclinaison dans la Programmation pluriannuelle de l'énergie $\left(\mathrm{PPE}^{99}\right)$ de 2016 ;

- par les engagements souscrits au niveau de l'Union européenne (« Paquet énergie-climat " de 2014) qui a défini trois objectifs à l'horizon 2030 pour l'Europe :

- une réduction de $40 \%$ des émissions de GES par rapport à 1990 ;

- une augmentation à $27 \%$ de la part des renouvelables dans la consommation énergétique finale brute

- en cours de révision, le Paquet énergie-climat pourrait porter de 27 à $30 \%$ la part des EnR en Europe et accentuer la réduction des émissions de GES, de la France en particulier, qui verrait son objectif porté à - $37 \%$ en 2030 par rapport à 2005 .

98. https://www.ccomptes.fr/fr/publications/le-cout-de-production-de-lelectricite-nucleaireactualisation-2014

99. https://www.ecologique-solidaire.gouv.fr/sites/default/files/PPE\%20int\%C3\%A9gralit\% C3\%A9.pdf 
La France s'est par ailleurs montrée très volontariste dans sa proposition de contribution (non engageante) dans le cadre des COP successives et en particulier la COP 21 de Paris en 2015. La priorité donnée désormais aux aspects environnementaux de la consommation d'énergie, induit deux grandes fragilités dans la vision énergétique future du pays, issue de la LTECV :

- d'une part notre pays pèse très peu dans les émissions mondiales (moins de $1 \%$ ) et s'est fixé, dans le cadre européen, des ambitions insoutenables, par exemple en acceptant pour 2030 par rapport à 2005 une baisse de ses émissions comparable à celle de l'Allemagne, alors que celle-ci émet $30 \%$ de plus de GES par habitant ${ }^{100}$. Les objectifs européens devraient veiller à une convergence des émissions de GES per capita en Europe, et non à imposer des réductions en fonction du seul PIB par habitant ;

- d'autre part l'impératif qu'il se fixe d'introduire une proportion croissante d'énergies renouvelables intermittentes (EnRi), éolienne et solaire essentiellement, dans la production électrique, associée à une diminution de la part de l'énergie nucléaire à $50 \%$, sera inefficace pour réduire les émissions de GES, car notre électricité est déjà décarbonée, et coûteux.

Cette réduction des émissions de GES en 2030 sera extrêmement difficile à respecter pour notre pays, voire inatteignable. Elle ne pourra en effet porter, pour l'essentiel, que sur les deux secteurs du transport et du résidentiel/tertiaire (voir Partie 2, Chapitre 3), et dans une bien moindre mesure sur l'industrie et l'agriculture. Il apparait clairement que le choix européen et français (dans la LTE et la PPE), d'imposer simultanément des obligations sur les moyens à mettre en ouvre pour atteindre l'objectif de limitation de la dérive climatique, peut être considéré comme un carcan qui s'oppose à une programmation efficace de cet objectif principal.

\subsection{Des éléments de réflexion pour une politique de l'énergie efficace et cohérente}

Il faudrait au préalable définir et classer les objectifs que l'on se donne pour cibles. Aujourd'hui, dans le contexte national mais aussi mondial, la limitation du réchauffement atmosphérique, soit une limitation à $2^{\circ} \mathrm{C}$ au plus de la température moyenne du globe d'ici la fin du siècle, est devenue fortement prioritaire, même si elle est déjà peu probable (la limitation à $1,5^{\circ} \mathrm{C}$ est déjà inenvisageable).

Réduire l'emploi des combustibles fossiles devient donc le premier but à atteindre, avant tous les autres objectifs techniques que sont la part d'EnR ou l'efficacité énergétique.

100. De ce fait, l'Allemagne aura plus de facilités à réaliser ses objectifs, par exemple en basculant du charbon et du lignite au gaz, ce que la France ne pourra faire. 


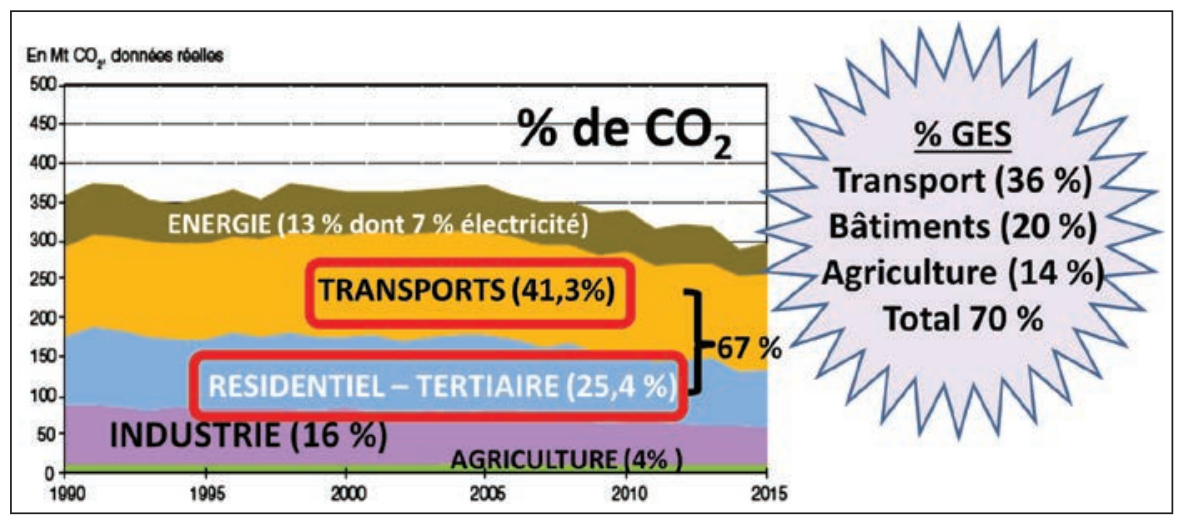

Figure 3 Les émissions de GES par secteurs en France en 2015.

Les secteurs d'activité concernés en priorité ressortent de la figure 3 : d'abord les transports (pétrole), puis le secteur résidentiel/tertiaire pour le chauffage et la climatisation (gaz et fioul).

Les moyens pour réduire les émissions de $\mathrm{CO}_{2}$ et celles des autres GES (CH4, $\mathrm{N} 2 \mathrm{O}, \mathrm{PFC}, \mathrm{HFC}, \mathrm{SF} 6$ ) sont très différents, mais en n'oubliant pas qu'en France le $\mathrm{CO}_{2}$ pèse pour environ les trois quarts dans l'impact climatique et le méthane pour $13 \%$.

- Les options pour réduire les émissions de $\mathrm{CO}_{2}$ sont diverses puisqu'on peut peser sur la consommation aussi bien que sur une substitution par des énergies non carbonées. Les aspects économiques et sociaux deviennent prépondérants pour définir un équilibre entre ces deux voies. La LTECV et la PPE actuelle ont donné priorité à une réduction de la part du nucléaire dans la production d'électricité, ce qui n'a pas de logique du point de vue climatique et résulte d'une position seulement idéologique.

Cette position présente pour le Gouvernement l'avantage d'être neutre sur un plan budgétaire car le soutien aux énergies renouvelables électrogènes passe par des « contributions » imposées aux clients (la CSPE et la CCE ${ }^{101}$ ) et des obliga-

101. L’ancienne contribution au service public de l'électricité est fusionnée avec la taxe intérieure sur la consommation finale d'électricité (TICFE), accise préexistante sur l'électricité qui reprend le nom de CSPE. Son niveau est fixé à 22,5 €/MWh pour 2016 et 2017. Les anciens plafonnements de CSPE disparaissent au profit de taux réduits pour certains types d'utilisation de l'électricité (installations industrielles électro-intensives, transport de personnes et de marchandises par train, métro, tramway, câble et trolleybus...).

Pour freiner la hausse de la CSPE, le développement des énergies renouvelables, jusqu'ici seulement financé par les consommateurs d'électricité, est désormais aussi supporté par les consommateurs d'énergie fossile (gaz, carburants...), au travers de la Contribution Climat Énergie (CCE) qui évoluera de $22 € / t$ en 2016 à $56 € /$ tonne de $\mathrm{CO}_{2}$ en 2020. 
tions d'achat par les distributeurs. Elle impose en parallèle des changements de mode de vie, qui dépendent essentiellement de la bonne volonté de la population, incertaine, et surtout d'une ambition démesurée, extrêmement coûteuse, de réduction des consommations.

- Les options pour réduire les émissions de $\mathrm{CH} 4$ seront d'une autre nature : réduction de l'élevage bovin et des consommations correspondantes, et gazéification des résidus agricoles.

Globalement la stratégie actuelle montre clairement ses limites avec des diminutions de consommation très faibles, d'un facteur 3 par rapport aux objectifs fixés:

- Le secteur résidentiel + tertiaire est fluctuant depuis dix ans, au niveau 66 à $68 \mathrm{Mtep}$, et baisse légèrement, de seulement $0,4 \%$ par an, depuis 3 ans (67 Mtep en 2015) ${ }^{102}$. La baisse restera lente car d'une part on ne construit que $1 \%$ du parc chaque année et d'autre part on ne rénove " thermiquement » que $1 \%$ des logements sans évaluation sérieuse des réductions de consommations effectives.

- Le secteur transports a baissé de $0,36 \%$ par an en 10 ans mais est en légère augmentation depuis 3 ans, soit + 0,6 \% par an (49,4 Mtep en 2015).

- Le secteur industriel a chuté de 2,2\% par an depuis 10 ans, y compris si on prend en compte le secteur non énergétique (pétrochimie par exemple). Sont principalement en cause la crise économique et la désindustrialisation du pays. La baisse se poursuit, de 1,6 \% par an depuis 3 ans (23,56 Mtep en 2015), mais pour le futur il faudra s'attacher à réindustrialiser le pays !

- Le secteur agricole et pêche a baissé de 3\% par an depuis 10 ans et de 1,3\% par an depuis 3 ans, mais reste très minoritaire, soit 4,45 Mtep en 2015.

Bien analyser l'expérience passée : face à cet échec du Grenelle de l'environnement aussi bien que de la LTECV, il est temps de revenir à une évaluation du rapport efficacité/coût économique de la politique mise en œuvre, mais aussi du réalisme des évolutions sociétales envisagées. Économies d'énergie, accroissement de l'efficacité énergétique, choix des filières, sont à peser rigoureusement et complètement, avec leurs coûts directs et indirects.

Il faut se méfier des idées toutes faites qui sont acceptées comme des vérités évidentes, du type " l'énergie la moins chère est celle que l'on ne dépense pas », «l'énergie du soleil et du vent sont gratuites »; le coût des investissements nécessaires à la réalisation d'une action (par exemple la réduction de consommation énergétique dans l'habitat) peut n'être jamais amorti par la baisse de la facture d'énergie consommée correspondante. Il faut aussi refuser les mesures dogmatiques telles que les normes pour le chauffage des bâtiments neufs (RT 2012) conçues pour favoriser le chauffage au gaz, émetteur de $\mathrm{CO}_{2}$, au détriment du chauffage électrique décarboné. Les bons marqueurs d'une vision énergétique du futur sont le prix de la tonne de GES évitée et l'efficacité d'usage de l'énergie finale.

102. L'ADEME indique une diminution de $1 \%$ par an depuis 5 ans, non vérifiée dans les statistiques officielles, et un report des énergies fossiles essentiellement vers le bois. 
Maintenir l'indépendance nationale vis-à-vis des sources et des technologies énergétiques demeure indispensable. Celle-ci plaide également en faveur d'une réduction du recours aux combustibles fossiles dont la France est dépourvue, en premier lieu du pétrole et du gaz. Sécurité et prix d'approvisionnement se trouvent en effet imprévisibles dans la durée (le prix du pétrole s'envole à nouveau après une période à bas coût qui avait permis à notre déficit commercial sur l'énergie de diminuer de 60 à 35 milliards en 5 ans). Parallèlement, le développement des EnR intermittentes que sont l'éolien et le solaire s'appuie sur des importations massives de matériels, alors même que le coût de l'énergie distribuée résulte principalement de l'investissement.

Recueillir l'assentiment de la société est un élément dont on ne peut plus se dispenser. On a vu le poids pris par les préoccupations environnementales et sociétales, souvent mises en avant par des groupes ou des associations actives dans la société. Il en est de même pour les problèmes de santé publique qui peuvent susciter une contestation, parfois violente ; c'est notamment le cas pour l'énergie nucléaire, pour les éoliennes et leurs nuisances visuelles et auditives, pour les gaz de schistes et leur atteinte aux aquiferes, etc.

L'Académie de médecine a ainsi tiré les conclusions d'un colloque sur les impacts sanitaires des différentes sources d'énergie. Elle montre, par exemple, que le charbon est celle qui entraîne le plus de décès par unité d'énergie produite et l'énergie nucléaire le moins. Une information équilibrée des citoyens doit revenir au premier plan des préoccupations du Gouvernement car elle ne peut être laissée à des ONG mondialisées et qui veulent imposer leurs idéologies, avec l'appui des médias.

Savoir sappuyer sur de réelles expertises : les organismes publics compétents et des instituts apportent souvent à ces questions des réponses reconnues pour leur qualité et leur indépendance, sans pour autant être écoutées. Tel est le cas pour l'Autorité de sûreté nucléaire quand on s'interroge sur la durée d'exploitation sûre des centrales nucléaires. En ce qui concerne la LTECV, les points de vue exprimés par de nombreux organismes ${ }^{103}$ sont clairs et bien argumentés. L'Académie des sciences a ainsi publié en 2017 une étude critique de la loi LTECV montrant les incohérences de certaines mesures concrètes face aux objectifs affichés ${ }^{104}$.

103. - Institut Montaigne, http://www.institutmontaigne.org/ressources/pdfs/publications/note_ energie_priorite_au_climat.pdf

- IFRAP : http://www.ifrap.org/sites/default/files/publications/fichiers/sc172_dossier_global_0.pdf

- FranceStratégie:http://www.strategie.gouv.fr//sites/strategie.gouv.fr/files/atoms/files/transition_ energetique_allemande_la_fin_des_ambitions_etienne_beeker_note_n59_aout_2017_0.pdf

- SLC : https://www.sauvonsleclimat.org/fr/base-documentaire

104. Académie des sciences : http://www.academie-sciences.fr/pdf/rapport/lpdv_190417.pdf 


\subsection{Conclusion}

Une politique de l'énergie, cohérente et robuste face aux évolutions du monde environnant, se bâtit nécessairement sur la longue durée. Or le temps politique et le temps industriel ne se déroulent pas au même rythme : un président de la République élu pour cinq ans, des investissements industriels réalisés pour cinquante ans et plus.

Il faudrait que les paramètres pris en compte dans les choix soient bien priorisés et clairs : par exemple, dans l'ordre, le coût de la tonne de $\mathrm{CO}_{2}$ évitée, le temps de retour sur les investissements consentis, la création d'emplois nationaux, l'évolution des prix pour les familles et l'économie.

L'impact global sur l'environnement d'une technologie doit bien sûr être évalué et comparé à celui de technologies concurrentes (y compris la pollution atmosphérique). Mais cela doit être fait en fonction de normes reconnues et non par application excessive d'un principe de précaution mal défini.

Les Gouvernements futurs devraient bénéficier de la souplesse d'action nécessaire à une bonne gouvernance dans un monde en évolution. Les objectifs techniques (taux d'EnR, niveaux de performances énergétiques des bâtiments par exemple) devraient être indicatifs et pilotés sur la base de plans glissants par chaque pays en fonction de ses atouts. Cette séparation des objectifs essentiels et des objectifs techniques devrait, au titre de la subsidiarité, être exigée de l'Europe, en opposition à la stratégie allemande, fondée sur une opposition à l'énergie nucléaire.

Il est donc indispensable qu'une stratégie énergétique soit construite sur des bases techniques et économiques solides et objectives, dans un esprit non partisan et non idéologique, après des débats ouverts dépassionnés. Ce sont des impératifs incontournables pour que les choix effectués contribuent à satisfaire efficacement les besoins et réduisent au maximum les risques liés aux incertitudes de l'avenir. 